\title{
GPCR-SSFE: A comprehensive database of G-protein-coupled receptor template predictions and homology models
}

\author{
Catherine L Worth ${ }^{1,2}$, Annika Kreuchwig ${ }^{1}$, Gunnar Kleinau ${ }^{1,3}$ and Gerd Krause ${ }^{1 *}$
}

\begin{abstract}
Background: G protein-coupled receptors (GPCRs) transduce a wide variety of extracellular signals to within the cell and therefore have a key role in regulating cell activity and physiological function. GPCR malfunction is responsible for a wide range of diseases including cancer, diabetes and hyperthyroidism and a large proportion of drugs on the market target these receptors. The three dimensional structure of GPCRs is important for elucidating the molecular mechanisms underlying these diseases and for performing structure-based drug design. Although structural data are restricted to only a handful of GPCRs, homology models can be used as a proxy for those receptors not having crystal structures. However, many researchers working on GPCRs are not experienced homology modellers and are therefore unable to benefit from the information that can be gleaned from such three-dimensional models. Here, we present a comprehensive database called the GPCR-SSFE, which provides initial homology models of the transmembrane helices for a large variety of family A GPCRs.

Description: Extending on our previous theoretical work, we have developed an automated pipeline for GPCR homology modelling and applied it to a large set of family A GPCR sequences. Our pipeline is a fragment-based approach that exploits available family A crystal structures. The GPCR-SSFE database stores the template predictions, sequence alignments, identified sequence and structure motifs and homology models for 5025 family A GPCRs. Users are able to browse the GPCR dataset according to their pharmacological classification or search for results using a UniProt entry name. It is also possible for a user to submit a GPCR sequence that is not contained in the database for analysis and homology model building. The models can be viewed using a Jmol applet and are also available for download along with the alignments.

Conclusions: The data provided by GPCR-SSFE are useful for investigating general and detailed sequencestructure-function relationships of GPCRs, performing structure-based drug design and for better understanding the molecular mechanisms underlying disease-associated mutations in GPCRs. The effectiveness of our multiple template and fragment approach is demonstrated by the accuracy of our predicted homology models compared to recently published crystal structures.
\end{abstract}

\section{Background}

Due to their fundamental role in signal transduction, $G$ protein-coupled receptors (GPCRs) are the target of a large proportion of medical drugs. GPCR structural data are important for understanding the molecular mechanisms underlying diseases caused by mutations in these receptors, as well as for structure-based drug design. Up until November 2010, experimentally determined three-

\footnotetext{
* Correspondence: gkrause@fmp-berlin.de

'Leibniz-Institut für Molekulare Pharmakologie (FMP), 13125 Berlin, Germany Full list of author information is available at the end of the article
}

dimensional structures of family A GPCRs were only available for four members - see Topiol and Sabio [1] for a review of these structures. This is in stark contrast to the total number of family A GPCRs, which for humans alone has recently been estimated at 750 receptors [2]. All of the family A GPCR structures share a common molecular architecture of seven transmembrane helices (TMHs), therefore the deficit in GPCR experimental structures can be rectified by building molecular models of GPCRs of unknown structure using homology modelling techniques [3]. Currently, there are

\section{Biomed Central}

C 2011 Worth et al; licensee BioMed Central Ltd. This is an Open Access article distributed under the terms of the Creative Commons Attribution License (http://creativecommons.org/licenses/by/2.0), which permits unrestricted use, distribution, and reproduction in any medium, provided the original work is properly cited. 
seven different GPCR structures to choose from when building homology models of GPCRs in the inactive state: bovine rhodopsin (Swiss-Prot:opsd_bovin) [4]; Japanese flying squid rhodopsin (Swiss-Prot:opsd_todpa) $[5,6]$; common turkey beta-1 adrenergic receptor (SwissProt:adrb1_melga) [7]; human beta-2 adrenergic receptor (Swiss-Prot:adrb2_human) [8,9]; human adenosine receptor A2A (Swiss-Prot:aa2ar_human) [10]; human dopamine D3 receptor (Swiss-Prot:drd3_human) [11] and human CXCR4 chemokine receptor (Swiss-Prot: cxcr4_human) [12]. However, despite the common general architecture of these structures, key differences do exist between them. The choice of which experimental GPCR structure(s) to use for building a comparative model of a particular GPCR is unclear and without detailed structural and sequence analyses, could be arbitrary.

In order to help clarify the choice of template selection for GPCR homology modelling, we recently developed a workflow for identifying the most appropriate template to use for building homology models of family A GPCRs [13]. We analyzed in detail conserved and unique sequence motifs and structural features in the five experimentally-determined GPCR structures available at the time. Our analysis provided deeper insight into specific and important structural features of GPCRs and valuable information for template selection. Using key features, we formulated a workflow for identifying the most appropriate template(s) for building homology models of GPCRs of unknown structure. Our analysis revealed that the available crystal structures represent only a subset of all possible structural variation in family A GPCRs. Some GPCRs have structural features that are distributed over different crystal structures or which are not present in the templates, suggesting that homology models should be built using a fragment approach whereby template selection is carried out for each $\mathrm{TMH}$, rather than the receptor as a whole.

There are a number of web resources available which focus on GPCRs. The IUPHAR database of GPCRs provides a comprehensive catalogue of peer-reviewed pharmacological, chemical, genetic, functional and anatomical information on human, rat and mouse nonsensory GPCRs, as well as links to other relevant resources [14]. The GPCRDB is an online information system for GPCRs which provides multiple sequence alignments, mutation data, ligand binding data and various tools such as an alignment builder and predicting the effects of mutations [15]. Interactions between human GPCRs, G proteins and effectors can be visualized using human-gpDB [16] whilst GPCR oligomer information is provided by GPCR-OKB [17]. Where structural data are provided by the aforementioned resources, they are limited to the handful of GPCRs with crystal structures available. The specialized GPCR databases SSFA [18] and GRIS [19] provide homology models of GPCRs, although these are restricted to the three glycoprotein hormone receptors. Zhang and coworkers used their threading assembler refinement method to build molecular models of 907 human GPCRs [20]. Although the models are available for download, they are currently not stored in a database and they were generated at a time when only the structure of bovine rhodopsin was available.

We have now extended our previous theoretical work and automated our workflow and applied it to a set of 5025 family A GPCR sequences. Based on these automated template suggestions, homology models were built of the seven TMHs and helix 8 of the 5025 receptors. Here, we present the GPCR-Sequence Structure Feature Extractor (SSFE) database, which stores the template predictions, the identified sequence and structure motifs and homology models of 5025 family A GPCRs. Users are able to browse the GPCR dataset stored in GPCR-SSFE or search for results using a UniProt entry name [21]. It is also possible for a user to submit a GPCR sequence that is not contained in the database for analysis, with the template suggestions for the seven TMHs and helix 8 returned along with a molecular model. The molecular models presented in GPCR-SSFE represent the most comprehensive set of GPCR models created so far and offer a valuable starting point for in silico functional predictions, docking studies and structure-based drug design.

\section{Construction and content GPCR dataset}

GPCR-SSFE contains all family A GPCRs sequences that were stored in the GPCRDB as of October 2009, totalling 5025 receptors [15].

\section{Template selection and homology modelling}

A profile hidden markov model (HMM) was used to align the 5025 GPCRs to the five template structures available at the time (before November 2010) (PDB codes: 1U19, 2Z73, 2VT4, 2RH1 and 3EML) [22]. HMMER2 http://hmmer.janelia.org/ was used to generate the profile HMM from a multiple sequence alignment (MSA) of the five templates plus 54 other family A GPCRs (see additional file 1 for a list of the GPCRs used to produce the HMM). The 54 GPCRs were selected so as to maximize the coverage of the phylogenetic tree for family A GPCRs [23]. After alignment, template selection was carried out for each TMH and helix 8 based on our previously published workflow [13], which for the purposes of this work was automated using python scripts. The workflow uses the presence or absence of structural features such as proline 
distortions, disulphide bridges and insertions as well as sequence similarity comparisons to select the best template(s) for modelling a given GPCR. Modeller 9v7 was used to generate homology models of query GPCRs using the alignments and templates suggested by GPCRSSFE [24]. Three models were produced for each GPCR but only the one with the lowest DOPE score [25] is stored in GPCR-SSFE.

\section{Database implementation}

GPCR-SSFE was built by combining an Apache web server http://www.apache.org, PHP Hypertext Pre-processor scripts (PHP 5) and a relational database management system (MySQL 5). The web interface is based on HTML, JavaScript and Cascading Style Sheets (CSS). The GPCR homology model structures are displayed using the Jmol structure viewer (Jmol: an open-source Java viewer for chemical structures in 3D http://www.jmol.org/.

\section{Utility}

\section{The GPCR-SSFE website}

The GPCR-SSFE has a user-friendly interface with a menu and a facts and questions (FAQ) page to aid usability. On the homepage of GPCR-SSFE (Figure 1), users can find information about the database and the template structures used for analysis and homology modelling, including a link to a Jmol applet showing the five template structures superimposed onto one another (Figure 2).

\section{Searching GPCR-SSFE}

Results can be retrieved by either browsing or searching the database. By navigating to the "Browse SSFE" menu option, the user is presented with a list of 19 family A GPCR subgroups (Figure 3A): Amine, Peptide, Hormone protein, (Rhod)opsin, Olfactory, Prostanoid, Nucleotidelike, Cannabinoid, Platelet activating factor, Gonadotropin-releasing hormone, Thyrotropin-releasing hormone \& Secretagogue, Melatonin, Viral, Lysophingolipid \& LPA (EDG), Leukotriene B4 receptors, Ecdysis triggering hormone receptor, Other, CAPA and Class A orphan/ other. These subgroups correspond to those used in the GPCRDB and are based on the pharmacological classification of GPCRs. Each subgroup can be expanded by clicking on it, revealing either further subgroupings or (if the final node is reached) a list of GPCRs within the subgroup. Clicking on the UniProt entry name of a GPCR will take the user to the results page for that particular receptor. Alternatively, users may retrieve results by entering the UniProt entry name onto the "Search SSFE" webpage (Figure 3B).

\section{GPCR-SSFE results page}

The results returned by GPCR-SSFE include (Figure 4): i) the templates used for analysis and links to the PDB
[26]; ii) the multiple sequence alignment (MSA) of the five templates and the query sequence for each individual transmembrane helix and helix eight; iii) the MSA of the profile HMM GPCR sequences and the query sequence, which spans the entire serpentine domain; iv) the HMMER2 e-value assigned to the full-length MSA; v) the template suggestions (and reasons) for the seven TMHs and helix eight; vi) the sequence similarity score between the suggested template(s) and the query sequence; vii) the rationale for the suggested templates; viii) a Jmol applet displaying the homology model(s) of the query GPCR based on the template suggestions; ix) the amino acid sequences of the intracellular and extracellular loops and $\mathrm{x}$ ) links to UniProt, the GPCRDB and, where applicable, to the Human-gpDB and GPCR-OKB databases. The loops are not modelled and therefore the loop sequences are provided to the user so that software such as SuperLooper may be used for modelling these regions [27]. Files containing results ii, iii, viii and ix are made available for download (Figure 4E). Additionally, for stereochemical quality checking a PROCHECK [28] generated Ramachandran plot of each model is provided and information about how to interpret the results are provided on the FAQ page.

\section{Running GPCR-SSFE}

In some instances, GPCR-SSFE might not store results for particular family A GPCRs e.g. newly identified orphan GPCRs. For such cases, users can submit their GPCR sequence (in FASTA format) to GPCR-SSFE for analysis and homology model building by navigating to the "Run SSFE" webpage (Figure 5). Modeller 9v7 is used for homology modelling, therefore, users must obtain a Modeller license key before they can use this tool. This key is freely available for academic users and easily obtainable at: http://salilab.org/modeller/registration.shtml. To start the template analysis and homology modelling, the user enters their GPCR sequence onto the webpage (by either uploading or copying and pasting it), along with their Modeller licence key and email address. Once the results are ready, a web-link is emailed to the user. Results are stored on the server for seven days. The results page looks exactly like those retrieved when searching the database, except that links to external databases are not provided.

\section{Performance: A case study}

Since completion of the large-scale GPCR model building and submission of the manuscript, two new family A GPCR crystal structures were published - the human dopamine D3 receptor (Swiss-Prot:drd3_human) [11] and the CXCR4 chemokine receptor (Swiss-Prot: cxcr4 human) [12]. These receptor structures therefore provide us with an ideal means of assessing the 


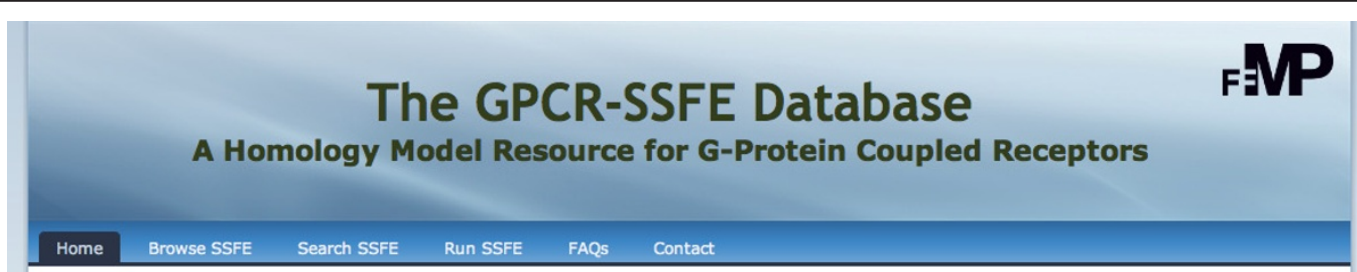

\section{- About GPCR-SSFE}

The GPCR-Sequence-Structure-Feature-Extractor (SSFE) database provides template suggestions and homology models of the helical regions of 5025 family A GPCRs. SSFE is based on our published workflow for identifying key sequence and structural motifs in family A GPCRs which is used to guide template selection and build homology models.

Users can access the models stored in GPCR-SSFE by either browsing the family (subdivided into 19 groups) or by searching for results using a UniProt identifier. Users can also enter a GPCR sequence of their choice for analysis by GPCR-SSFE. Results returned include the suggestion of which templates to use for modelling, sequence alignments. and homology models of the predicted helical regions of the query GPCR.

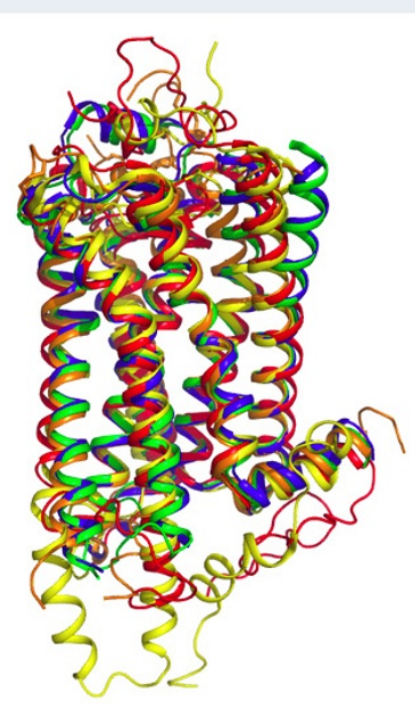

\section{Structural superimposition of the five available GPCR structures}

The overall topology of the templates is remarkably similar, with the transmembrane helices superimposing relatively well in most cases. hAA2AR (PDB entry 3EML 7 ) is represented in orange, tB1AR in blue (PDB entry 2VT4 ), hB2AR in green (PDB entry 2RH1v), sRho in yellow (PDB entry 2Z73v) and bRho in red (PDB entry 1U19v). The image was rendered using Pymol.

Please click here to view the five superimposed GPCR structures in 3D using Jmol.

\section{- References}

The methodology used for template selection is detailed in the following paper:

Worth CL, Kleinau G and Krause G (2009) Comparative sequence and structural analyses of G-protein-coupled receptor crystal structures and implications for molecular models. PLoS One 4(9): e7011.

\section{Disclaimer/Citation I Contributors I Lab Gerd Krause}

Last updated: 20 August 2010

Figure 1 The home page of the GPCR-SSFE database. The menu at the top of the page allows users to easily navigate the site. The background to the database is provided, along with information about the templates used for homology modelling.

performance of the models produced by GPCR-SSFE as they were not included in the pool of crystal structure templates used by GPCR-SSFE and we can now calculate the RMSD of our predicted models to the crystal structures. In order to assess the impact of increased template similarity on the accuracy of the homology models we used different sets of templates for modelling, based on their sequence similarity across the entire serpentine domain:

1) Bovine rhodopsin (PDB:1U19) and Japanese flying squid rhodopsin (PDB:2Z73

2) $1 \mathrm{U} 19,2 \mathrm{Z} 73$ and human Adenosine receptor A2A (PDB:3EML) 


\section{- 3D Superimposition of the five template GPCRs}

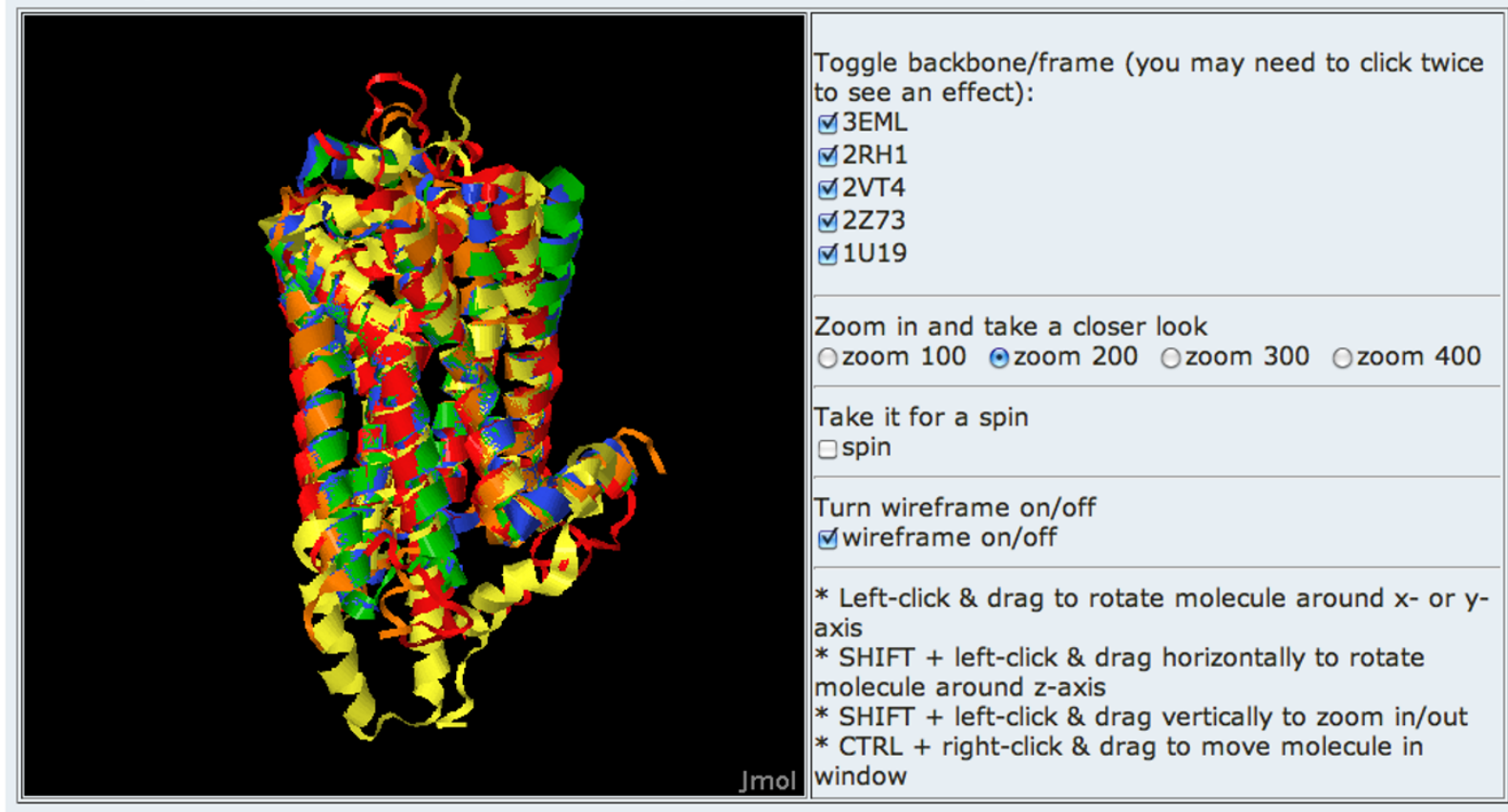

Figure 2 Structural superimposition of the GPCR template structures. Users can access a Jmol applet displaying the five crystal structures used for analysis and homology modelling via the home page.

3) $1 \mathrm{U} 19,2 \mathrm{Z73}, 3 \mathrm{EML}$, common turkey Beta-1 adrenergic receptor (PDB;2VT4) and human Beta-2 adrenergic receptor (PDB:2RH1)

In order to assess the impact of using multiple templates for building homology models compared to using the single most similar template, we also built a model for each of the two GPCRs using only the GPCR structure with the highest sequence similarity across the entire serpentine domain, which in both cases was:

\section{4) $2 \mathrm{VT} 4$}

5) Finally, we used I-TASSER [29], which was ranked 1 in the server category of the Critical Assessment of Structure Prediction (CASP) 20072009, to build a model of both drd3_human and cxcr4_human, excluding their respective crystal structures from template selection.

For steps 1 to 4, three models were produced using Modeller 9v7 with the model having the lowest DOPE score selected for further accuracy analysis. I-TASSER produces five models and provides a confidence score (C-score) to indicate the likely best model. Therefore, we chose the I-TASSER model with the highest C-score for further accuracy analysis.
The accuracy of the models generated using the different template sets and methods above were assessed by calculating the RMSD between the $\mathrm{C}-\alpha$ residues in the transmembrane region using the align feature of Pymol (Table 1). Unsurprisingly, the results indicate that where multiple templates are used, the addition of more similar templates improves the models e.g. models produced using the two rhodopsin templates (lowest sequence similarity templates to the two targets) had higher RMSDs than the models produced using all five templates. The use of multiple templates compared to a single template produces fairly similar RMSDs, although the multiple template model produced for drd3_human is slightly better than that produced using the single closest template $(0.88 \AA$ compared to $0.94 \AA)$. Furthermore, where the full number of templates are used for modelling with GPCR-SSFE, the model produced for drd3_human is similar in accuracy to that produced by ITASSER and better than I-TASSER in the case of cxcr4_human.

We also did a comparable analysis of the five template structures and observed similar results to those observed for drd3_human and cxcr4_human, although in some instances the models produced by I-TASSER are significantly worse due to part of the transmembrane domain being modelled on the T4 lysozyme insertion found in 


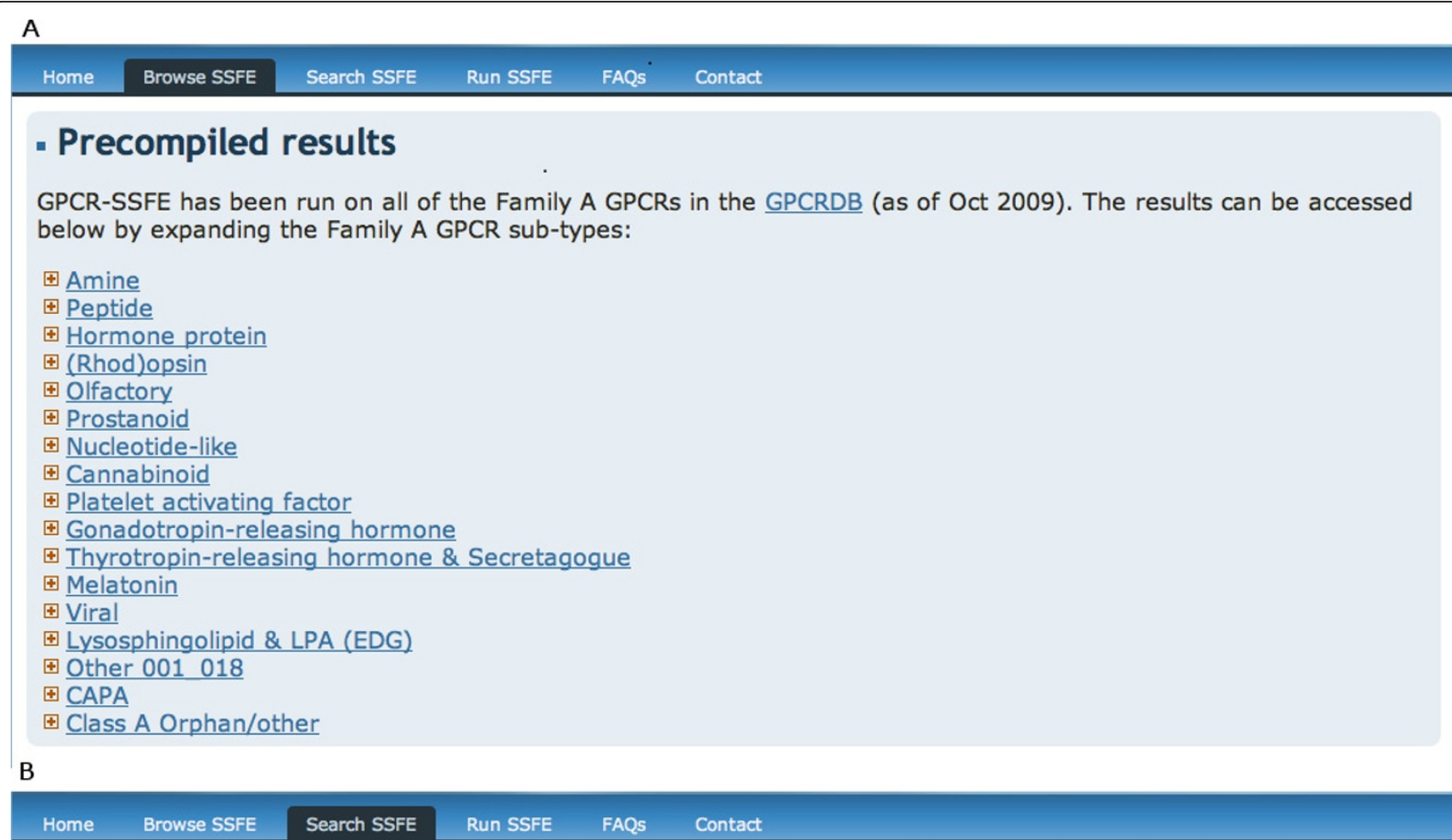

\section{- Search for Precompiled Results}

Query the GPCR-SSFE database by a given Uniprot entry name

Uniprot Entry name e.g. acm1_human

Next

Figure 3 Querying the GPCR-SSFE database. Results may be retrieved from the database by either A) browsing the database via a list of 19 family A GPCR subgroups or B) by searching using a UniProt entry name.

some GPCR crystal structures (See additional file 2, Tables S1-S5).

These results validate the effectiveness of the multiple template and fragment approach to homology model building employed by SSFE and indicate that the models are as accurate and, in some cases, better than those produced by one of the current best performing homology modelling programs (as measured by the CASP [30]). We plan to incorporate these two new structures to GPCRSSFE's workflow and pool of available templates in the very near future and then re-run the template analysis and model building for the 5025 GPCRs in the database.

Our results show that the addition of more similar templates improves the quality of the models and that the single template generated models are similar or slightly worse than the multiple template models. Finally, our results demonstrate that the models produced for the human CXCR4 chemokine receptor and the human dopamine D3 receptor are native like, having RMSDs compared to their crystal structures of 1.7 and 0.9 respectively.

\section{Discussion}

A recent study demonstrated that automated modelling of human neurokinin-1 receptor was enriched by a factor of 2.6 when a combination of bovine rhodopsin and beta-2 adrenergic receptor were used as templates rather than when used singly [31]. Similarly, Mobarec and coworkers found that the use of multiple templates can provide similar or slightly improved models of the transmembrane regions of GPCRs compared to receptor models obtained using single templates [32]. Although a modelling study on opioid receptors demonstrated that it is not always true that a GCPR homology model built using multiple templates is structurally better than those built using one [33]. 


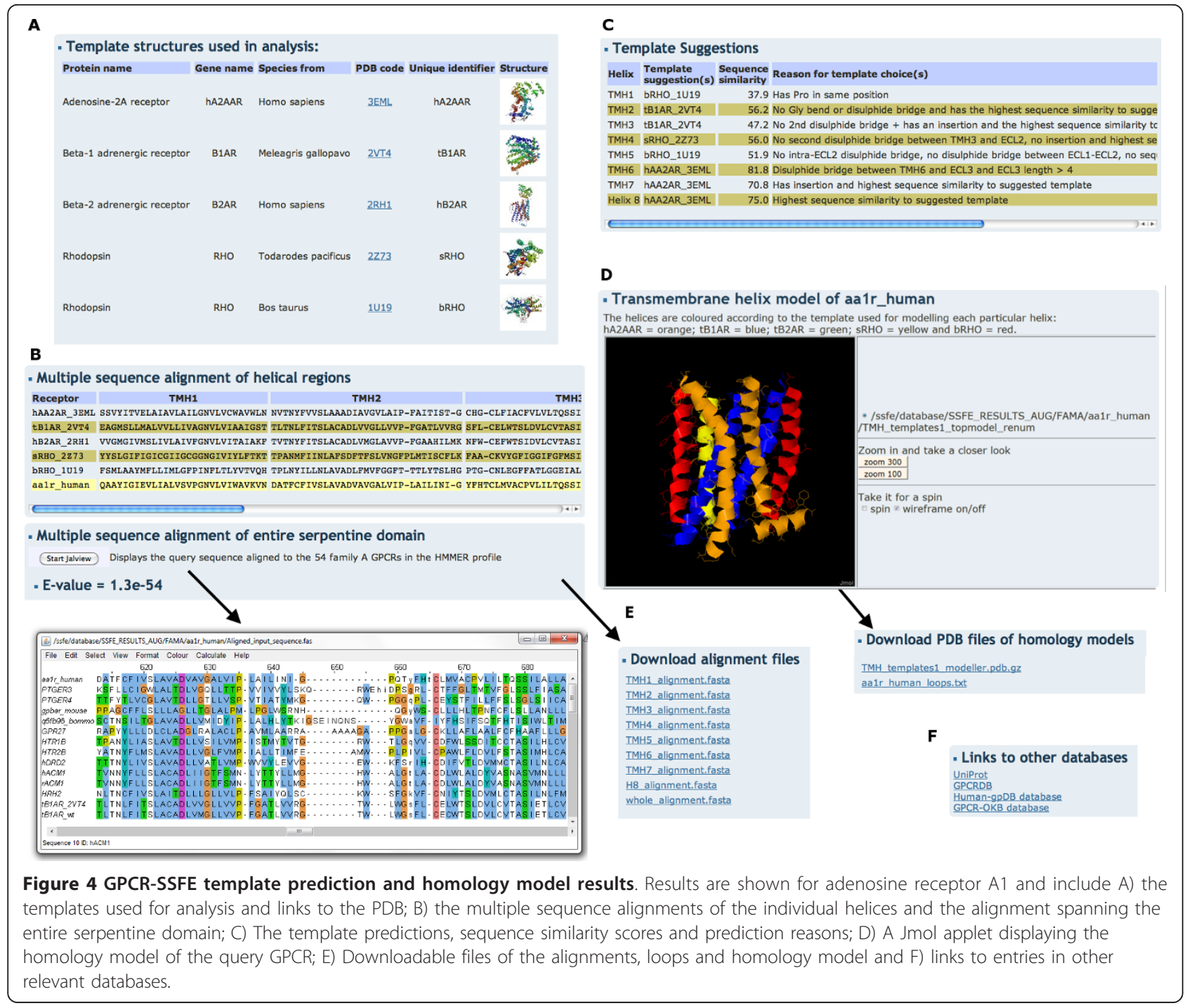

Our approach to building homology models of GPCRs has a number of advantages over other recent similar studies. Firstly, GPCR-SSFE uses all available family A GPCR templates (that were available at the time of performing the work) to estimate all of the sequence related structural features for a particular receptor and to generate an optimal GPCR model. Secondly, the template for each transmembrane helix is selected individually, meaning that either a single template or a fragment-based approach can be used for model building. This is an important feature considering that it has been shown that sometimes models that are built using one template are better than those built using multiple templates, whereas at other times multiple template models are better [31,32]. Thirdly, GPCR-SSFE has been applied to a very large set of family A GPCRs and all of the homology models have been made available for download and stored in a database. The availability of these initial models allows researchers who are not experienced homology modellers to access three-dimensional data of GPCRs that they are interested in. The user should be aware however, that some GPCR sequences lack particular motifs that are responsible for structural features and which currently occur in all of the available templates, for example, the helix bulges caused by prolines in TMH 5. Since the prolines and bulges are present in all of the templates, they also subsequently occur in the homology models, even when a proline is not present in the corresponding position in a target sequence. Thus, in such cases further refinement of the initial model may be required.

Due to their variability in sequence, length and conformational flexibility, the loop regions of GPCRs are far more difficult to predict by both homology modelling and de novo modelling techniques $[34,35]$. Based on their dockings studies on human beta- 2 adrenergic 


\section{\begin{tabular}{lllll} 
Home & Browse SSFE & Search SSFE & Run SSFE & FAQS \\
\hline
\end{tabular}}

\section{- Submit a GPCR sequence for analysis by GPCR-SSFE}

This requires you to have obtained a license key to run MODELLER! If you do not have a MODELLER license key then please register at http://salilab.org/modeller/registration.

\section{Please submit your Family A GPCR for analysis by either:}

A. Copying and pasting its sequence (in FASTA format) in the box below:

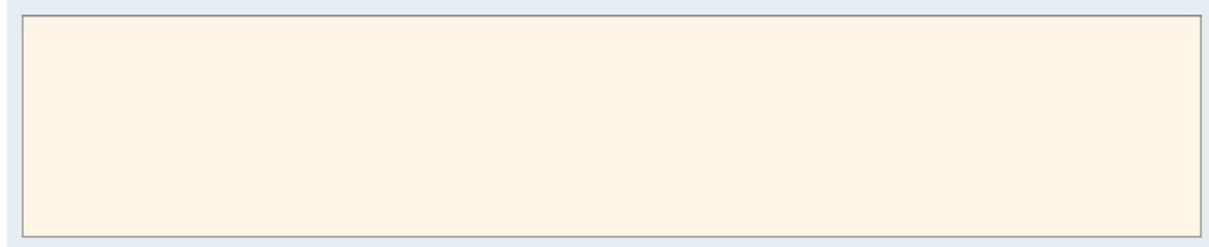

B. Uploading a file containing its sequence (in FASTA format) using the box below:

Choose a file to upload: Choose file no file selected (maximum file size $=100 \mathrm{~KB}$ )

2. Please enter your email address so that the results can be sent to you:

Please note: We do not keep a record of user's email addresses.

Pase note: We do not keep a record of user's

3. Please enter your MODELLER license key:

Submit GPCR sequence

Figure 5 GPCR-SSFE sequence submission page. Where a family A GPCR is not contained in the database, a user may submit their sequence of interest to GPCR-SSFE for template prediction and homology modelling. Users must obtain a licence key for Modeller before they are able to use this function.

receptor, Mobarec and coworkers speculate that GPCR models without loops may constitute a better alternative for flexible ligand-rigid protein docking than those with loops [32]. Therefore, although the models built using GPCR-SSFE are loopless, we are confident that they will be extremely useful for i) investigating the mechanisms underlying structural and/or functional effects of mutations; ii) performing docking studies and iii) structurebased drug design. Nevertheless, the provided initial models can be completed by building the loops with suitable software tools [20].

\section{Conclusions}

Our previous analysis indicates that, in general, the structural features of target GPCRs cannot be captured using only one of the experimental GPCR structures as a template for homology modelling. Consequently, we suggest that using transmembrane fragments from

Table 1 The RMSD of cxcr4_human and drd3_human homology models compared to their crystal structures

\begin{tabular}{|c|c|c|c|}
\hline Method used for template selection & Templates used & $\begin{array}{l}\text { Accuracy cxcr4_human } \\
\text { (RMSD) }^{1}\end{array}$ & $\begin{array}{l}\text { Accuracy drd3_human } \\
\text { (RMSD) }^{2}\end{array}$ \\
\hline $\begin{array}{l}\text { Sequence similarity across entire serpentine } \\
\text { domain }\end{array}$ & 2VT4 & 1.72 & 0.94 \\
\hline Sequence similarity for each TMH & $1 \cup 19$ and $2 Z 73$ & 1.78 & 1.21 \\
\hline $\begin{array}{l}\text { SSFE workflow or sequence similarity for each } \\
\text { TMH }\end{array}$ & $1 \mathrm{U} 19,2 \mathrm{Z73}$ and $3 \mathrm{EML}$ & 1.97 & 1.04 \\
\hline SSFE workflow & $1 \mathrm{U} 19,2 \mathrm{Z73}, 3 \mathrm{EML}, 2 \mathrm{VT} 4$ and $2 \mathrm{RH} 1$ & 1.73 & 0.88 \\
\hline I-TASSER & $\begin{array}{l}\text { Crystal structure of query protein } \\
\text { excluded }\end{array}$ & 1.91 & 0.82 \\
\hline
\end{tabular}

${ }^{1}$ RMSD between cxcr4_human model and 3ODU (TMH region)

${ }^{2}$ RMSD between drd3_human model and 3PBL (TMH region) 
multiple templates when building comparative models of GPCRs is likely to lead to more accurate results, an approach that has also been advocated by other published studies on molecular modelling of GPCRs $[3,20]$. GPCR-SSFE is the first comprehensive GPCR-focused database to store homology models based on the crystallographic advances made in recent years. The fragmentbased models stored in GPCR-SSFE serve as a valuable starting-point for molecular analyses of GPCRs based on their structure, such as structure-based drug design or the detailed molecular analysis of mutations. We believe that GPCR-SSFE will be of increasing importance as more crystal structures become available because the problem of template selection will become more complex.

\section{Availability and requirements Availability}

GPCR-SSFE is freely available for use on the web at: http://www.ssfa-7tmr.de/ssfe/. The database is publicly and freely accessible, requiring no registration and with no restrictions on use. The GPCR-SSFE server tool requires users to have a Modeller license key, which can be easily and quickly obtained by academic users at the Modeller website http://www.salilab.org/modeller/registration.html.

\section{Technical requirements}

GPCR-SSFE requires that the web browser used supports JavaScript and CSS and that Java is installed and working. It is recommended that one of the following browsers is used: Mozilla Firefox 3 on Linux, Mac OSX or Windows, Internet Explorer 8 on Windows, Safari 4 on Mac OXS or Windows, Chrome on Linux or Windows.

\section{Additional material}

Additional file 1: List of the GPCRs in the HMMER profile used for alignment. List of GPCRs used to build the HMMER profile used for sequence alignment by GPCR-SSFE. The identifier used in the alignment, UniProt entry name, phylogenetic group and cluster (according to the phylogenetic tree for family A [23]) and the receptor type are given. The five GPCRs with crystal structures are highlighted in grey. The amino acid sequences of 3EML, 2VT4 and 2RH1 were modified compared to wildtype (due to either substitutions or deletions) and therefore the wildtype sequences were also included in the profile.

Additional file 2: Accuracy analysis for the five template structures. Contains the RMSD values for the models produced using the single most similar template, multiple templates of increasing similarity and ITASSER.

\section{Abbreviations}

CASP: Critical Assessment of Structure Prediction; CSS: cascading style sheets; FAQ: Facts and Questions; GPCR: G protein-coupled receptor; HMM: Hidden Markov Model; HTML: Hypertext Markup Language; LPA: lysophosphatidic acid; MSA: multiple sequence alignment; PDB: Protein Data Bank; TMH: transmembrane helix; RMSD: Root Mean Squared Deviation

\section{Acknowledgements}

This work was supported by a DAAD Kurt Hahn research grant to CLW.

\section{Author details}

${ }^{1}$ Leibniz-Institut für Molekulare Pharmakologie (FMP), 13125 Berlin, Germany. ${ }^{2}$ Structural Bioinformatics Group, Charité - Universitätsmedizin, 13125 Berlin, Germany. ${ }^{3}$ Institut für Experimentelle Pädiatrische Endokrinologie, Charité Universitätsmedizin, Augustenburgerplatz 1, 13353 Berlin, Germany.

\section{Authors' contributions}

CLW performed the algorithm development, homology modelling, designed the database, developed the web interface and wrote the manuscript. AK implemented the search function. CLW and GKr conceived of the study. AK, $\mathrm{GKr}$ and $\mathrm{GKI}$ conducted the testing. All the authors read and approved the final manuscript.

Received: 22 September 2010 Accepted: 23 May 2011

Published: 23 May 2011

\section{References}

1. Topiol S, Sabio M: X-ray structure breakthroughs in the GPCR transmembrane region. Biochem Pharmacol 2009, 78:11-20.

2. Fredriksson $\mathrm{R}$, Schioth HB: The repertoire of G-protein-coupled receptors in fully sequenced genomes. Mol Pharmacol 2005, 67:1414-25.

3. Yarnitzky T, Levit A, Niv MY: Homology modeling of G-protein-coupled receptors with X-ray structures on the rise. Curr Opin Drug Discov Devel 2010, 13:317-25.

4. Palczewski K, Kumasaka T, Hori T, Behnke CA, Motoshima H, Fox BA, Trong Le I, Teller DC, Okada T, Stenkamp RE, Yamamoto M, Miyano M: Crystal structure of rhodopsin: A G protein-coupled receptor. Science 2000, 289:739-45.

5. Murakami M, Kouyama T: Crystal structure of squid rhodopsin. Nature 2008, 453:363-7.

6. Shimamura T, Hiraki K, Takahashi N, Hori T, Ago H, Masuda K, Takio K, Ishiguro M, Miyano M: Crystal structure of squid rhodopsin with intracellularly extended cytoplasmic region. J Biol Chem 2008, 283:17753-6.

7. Warne T, Serrano-Vega MJ, Baker JG, Moukhametzianov R, Edwards PC Henderson R, Leslie AG, Tate CG, Schertler GF: Structure of a beta1adrenergic G-protein-coupled receptor. Nature 2008, 454:486-91.

8. Cherezov V, Rosenbaum DM, Hanson MA, Rasmussen SG, Thian FS, Kobilka TS, Choi HJ, Kuhn P, Weis WI, Kobilka BK, Stevens RC: Highresolution crystal structure of an engineered human beta2-adrenergic $\mathrm{G}$ protein-coupled receptor. Science 2007, 318:1258-65.

9. Rasmussen SG, Choi HJ, Rosenbaum DM, Kobilka TS, Thian FS, Edwards PC, Burghammer M, Ratnala VR, Sanishvili R, Fischetti RF, Schertler GF, Weis WI, Kobilka BK: Crystal structure of the human beta2 adrenergic G-proteincoupled receptor. Nature 2007, 450:383-7.

10. Jaakola VP, Griffith MT, Hanson MA, Cherezov V, Chien EY, Lane JR, Ijzerman AP, Stevens RC: The 2.6 angstrom crystal structure of a human A2A adenosine receptor bound to an antagonist. Science 2008, 322:1211-7.

11. Chien EY, Liu W, Zhao Q, Katritch V, Han GW, Hanson MA, Shi L, Newman AH, Javitch JA, Cherezov V, Stevens RC: Structure of the human dopamine D3 receptor in complex with a D2/D3 selective antagonist. Science 2010, 330:1091-5.

12. Wu B, Chien EY, Mol CD, Fenalti G, Liu W, Katritch V, Abagyan R, Brooun A, Wells P, Bi FC, Hamel DJ, Kuhn P, Handel TM, Cherezov V, Stevens RC: Structures of the CXCR4 chemokine GPCR with small-molecule and cyclic peptide antagonists. Science 2010, 330:1066-71.

13. Worth $\mathrm{CL}$, Kleinau G, Krause G: Comparative sequence and structural analyses of G-protein-coupled receptor crystal structures and implications for molecular models. PLoS One 2009, 4:e7011.

14. Harmar AJ, Hills RA, Rosser EM, Jones M, Buneman OP, Dunbar DR, Greenhill SD, Hale VA, Sharman JL, Bonner TI, Catterall WA, Davenport AP, Delagrange P, Dollery CT, Foord SM, Gutman GA, Laudet V, Neubig RR, Ohlstein EH, Olsen RW, Peters J, Pin JP, Ruffolo RR, Searls DB, Wright MW, Spedding M: IUPHAR-DB: the IUPHAR database of G protein-coupled receptors and ion channels. Nucleic Acids Res 2009, 37:D680-5. 
15. Horn F, Weare J, Beukers MW, Horsch S, Bairoch A, Chen W, Edvardsen O, Campagne F, Vriend G: GPCRDB: an information system for G proteincoupled receptors. Nucleic Acids Res 1998, 26:275-9.

16. Satagopam VP, Theodoropoulou MC, Stampolakis CK, Pavlopoulos GA, Papandreou NC, Bagos PG, Schneider R, Hamodrakas SJ: GPCRs, G-proteins, effectors and their interactions: human-gpDB, a database employing visualization tools and data integration techniques. Database (Oxford) 2010, 2010:baq019.

17. Khelashvili G, Dorff K, Shan J, Camacho-Artacho M, Skrabanek L, Vroling B, Bouvier M, Devi LA, George SR, Javitch JA, Lohse MJ, Milligan G, Neubig RR, Palczewski K, Parmentier M, Pin JP, Vriend G, Campagne F, Filizola M: GPCROKB: the G Protein Coupled Receptor Oligomer Knowledge Base. Bioinformatics 2010, 26:1804-5.

18. Kleinau G, Kreuchwig A, Worth $C L$, Krause G: An interactive web-tool for molecular analyses links naturally occurring mutation data with threedimensional structures of the rhodopsin-like glycoprotein hormone receptors. Hum Mutat 2010, 31:E1519-25.

19. Van Durme J, Horn F, Costagliola S, Vriend G, Vassart G: GRIS: glycoproteinhormone receptor information system. Mol Endocrinol 2006, 20:2247-55.

20. Zhang Y, Devries ME, Skolnick J: Structure modeling of all identified G protein-coupled receptors in the human genome. PLoS Comput Biol 2006, 2:e13.

21. The UniProt Consortium: The Universal Protein Resource (UniProt) in 2010. Nucleic Acids Res 2010, 38:D142-8.

22. Durbin R, Eddy S, Krogh A, Mitchison G: The theory behind profile HMMs. Biological sequence analysis: probabilistic models of proteins and nucleic acids Cambridge: Cambridge University Press; 1998.

23. Fredriksson R, Lagerstrom MC, Lundin LG, Schioth HB: The G-proteincoupled receptors in the human genome form five main families. Phylogenetic analysis, paralogon groups, and fingerprints. Mol Pharmacol 2003, 63:1256-72.

24. Sali A, Blundell TL: Comparative protein modelling by satisfaction of spatial restraints. J Mol Biol 1993, 234:779-815.

25. Shen MY, Sali A: Statistical potential for assessment and prediction of protein structures. Protein Sci 2006, 15:2507-24.

26. Berman HM, Westbrook J, Feng Z, Gilliland G, Bhat TN, Weissig H, Shindyalov IN, Bourne PE: The Protein Data Bank. Nucleic Acids Res 2000, 28:235-42.

27. Hildebrand PW, Goede A, Bauer RA, Gruening B, Ismer J, Michalsky E, Preissner R: SuperLooper-a prediction server for the modeling of loops in globular and membrane proteins. Nucleic Acids Res 2009, 37:W571-4.

28. Laskowski RA, MacArthur MW, Moss DS, Thornton JM: PROCHECK - a program to check the stereochemical quality of protein structures. J. App. Cryst 1993, 26:283-91.

29. Zhang Y: I-TASSER server for protein 3D structure prediction. BMC Bioinformatics 2008, 9:40.

30. Zhang Y: I-TASSER: fully automated protein structure prediction in CASP8. Proteins 2009, 77(Suppl 9):100-13.

31. Kneissl B, Leonhardt B, Hildebrandt A, Tautermann CS: Revisiting automated G-protein coupled receptor modeling: the benefit of additional template structures for a neurokinin-1 receptor model. J Med Chem 2009, 52:3166-73.

32. Mobarec JC, Sanchez R, Filizola M: Modern Homology Modeling of GProtein Coupled Receptors: Which Structural Template to Use? J Med Chem 2009, 52(16):5207-16.

33. Bera I, Laskar A, Ghoshal N: Exploring the structure of opioid receptors with homology modeling based on single and multiple templates and subsequent docking: A comparative study. J Mol Model 2010, Epub ahead of print.

34. Michino M, Abola E, Brooks CL, Dixon JS, Moult J, Stevens RC: Communitywide assessment of GPCR structure modelling and ligand docking: GPCR Dock 2008. Nat Rev Drug Discov 2009, 8:455-63.

35. Michino M, Chen J, Stevens RC, Brooks CL: FoldGPCR: structure prediction protocol for the transmembrane domain of $\mathrm{G}$ protein-coupled receptors from class A. Proteins 2010, 78:2189-201.

doi:10.1186/1471-2105-12-185

Cite this article as: Worth et al: GPCR-SSFE: A comprehensive database of G-protein-coupled receptor template predictions and homology models. BMC Bioinformatics 2011 12:185.

\section{Submit your next manuscript to BioMed Central and take full advantage of:}

- Convenient online submission

- Thorough peer review

- No space constraints or color figure charges

- Immediate publication on acceptance

- Inclusion in PubMed, CAS, Scopus and Google Scholar

- Research which is freely available for redistribution 